\title{
Title: Phenome-wide association studies (PheWAS) across large "real-world data" population cohorts support drug target validation
}

Authors: Dorothée Diogo ${ }^{1}$, Chao $\operatorname{Tian}^{2}$, Christopher S. Franklin ${ }^{3}$, Mervi Alanne-Kinnunen ${ }^{4}$, Michael March $^{5}$, Chris C. A. Spencer ${ }^{3}$, Ciara Vangjeli ${ }^{3}$, Michael E. Weale ${ }^{3}$, Hannele Mattsson ${ }^{4,6}$, Elina Kilpeläinen ${ }^{4}$, Patrick M.A. Sleiman ${ }^{5}$, Dermot F. Reilly ${ }^{1}$, Joshua McElwee ${ }^{1}$, Joseph C. Maranville ${ }^{1}$, Arnaub K Chatterjee $^{1} \dagger$, Aman Bhandari ${ }^{1}+$, the 23 andMe Research Team ${ }^{2}$, Mary-Pat Reeve ${ }^{7}$, Janna Hutz ${ }^{7}$, Nan Bing ${ }^{8}$, Sally John ${ }^{9}$, Daniel G. MacArthur ${ }^{10,11}$, Veikko Salomaa ${ }^{6}$, Samuli Ripatti ${ }^{4}$, Hakon Hakonarson ${ }^{5}$, Mark J. Daly $^{10,11}$, Aarno Palotie ${ }^{4,10,11,12,13}$, David Hinds ${ }^{2}$, Peter Donnelly ${ }^{3}$, Caroline S. Fox ${ }^{1}$, Aaron Day-Williams ${ }^{1,9}$, Robert M. Plenge ${ }^{1} \S$, and Heiko Runz ${ }^{1}$ *

\section{Affiliations:}

${ }^{1}$ Merck Sharp \& Dohme, Kenilworth, NJ, USA.

2 23andMe Inc., Mountain View, CA, USA.

${ }^{3}$ Genomics Plc, Oxford, UK.

${ }^{4}$ Institute for Molecular Medicine Finland (FIMM), University of Helsinki, Helsinki, Finland.

${ }^{5}$ The Children's hospital of Philadelphia and University of Pennsylvania, Philadelphia, PA, USA.

${ }^{6}$ National Institute for Health and Welfare, Helsinki, Finland.

${ }^{7}$ Eisai, Andover, MA, USA.

${ }^{8}$ Pfizer, Cambridge, MA, USA.

${ }^{9}$ Biogen, Research and Early Development, Cambridge, MA, USA.

${ }^{10}$ Broad Institute of MIT and Harvard, Cambridge, MA, USA.

${ }^{11}$ Analytic and Translational Genetics Unit, Department of Medicine, Massachusetts General Hospital, Boston, MA, USA. 

not certified by peer review) is the author/funder. All rights reserved. No reuse allowed without permission.

${ }^{12}$ Psychiatric \& Neurodevelopmental Genetics Unit, Department of Psychiatry, Massachusetts General Hospital, Boston, MA, USA.

${ }^{13}$ Department of Neurology, Massachusetts General Hospital, Boston, MA, USA.

$\dagger$ Current address: McKinsey \& Co., Boston, MA, USA.

† Current address: Vertex Pharmaceuticals, Boston, MA, USA.

$\S$ Current address: Celgene, Cambridge, MA, USA.

* Correspondence to: heiko.runz@gmail.com 


\title{
One Sentence Summary:
}

Matching genetics with phenotypes in 800,000 individuals predicts efficacy and on-target safety of future drugs.

\begin{abstract}
:
Phenome-wide association studies (PheWAS), which assess whether a genetic variant is associated with multiple phenotypes across a phenotypic spectrum, have been proposed as a possible aid to drug development through elucidating mechanisms of action, identifying alternative indications, or predicting adverse drug events (ADEs). Here, we evaluate whether PheWAS can inform target validation during drug development. We selected 25 single nucleotide polymorphisms (SNPs) linked through genome-wide association studies (GWAS) to 19 candidate drug targets for common disease therapeutic indications. We independently interrogated these SNPs through PheWAS in four large "real-world data" cohorts (23andMe, UK Biobank, FINRISK, CHOP) for association with a total of 1,892 binary endpoints. We then conducted meta-analyses for 145 harmonized disease endpoints in up to 697,815 individuals and joined results with summary statistics from 57 published GWAS. Our analyses replicate 70\% of known GWAS associations and identify 10 novel associations with study-wide significance after multiple test correction $\left(\mathrm{P}<1.8 \times 10^{-6}\right.$; out of 72 novel associations with FDR $<0.1$ ). By leveraging directionality and point estimate of the effect sizes, we describe new associations that may predict ADEs, e.g., acne, high cholesterol, gout and gallstones for rs738409 (p.I148M) in PNPLA3; or asthma for rs1990760 (p.T946A) in IFIH1. We further propose how quantitative estimates of genetic safety/efficacy profiles can be used to help prioritize candidate targets for a specific indication. Our results demonstrate PheWAS as a powerful addition to the toolkit for drug discovery.
\end{abstract}




\section{[Main Text:]}

\section{INTRODUCTION}

The discovery and development of novel therapeutics is difficult. It may take 15 years to advance a new molecular entity from therapeutic hypothesis to approval, with development costs in the billion dollar range and only $10 \%$ chance of a new drug tested in humans eventually getting approval $^{1}$. Two reasons stand out to explain the high failure rate of clinical trials and receding return on R\&D investment across the pharmaceutical industry: A lower efficacy of the compound in the targeted disease population than anticipated from preclinical studies; and the occurrence of unintended drug effects, particularly mechanism-based adverse drug events (ADEs) uncovered only in late-stage clinical trials ${ }^{2}$. A greater understanding of human data relevant to the drug target at early stages of drug development is generally considered to increase the probability of success ${ }^{1}$, 3,4

Resources that systematically capture biomedical information on vast numbers of individuals are revolutionizing our ability to understand the complexities of human biology and morbidity. Electronic health records (EHRs) and similar "Real-World Data" (RWD) have rapidly become well-established tools for epidemiological and post-marketing research ${ }^{5,6}$. Recently, a surge of initiatives have sought to link such phenotype resources with genome-scale genetic data in order to gain further insights into the genetics of common diseases $7,8,9,10,11,12,13,14$.

An attractive approach for such genotype-phenotype resources to help accelerate drug development is through Phenome-Wide Association Studies (PheWAS). PheWAS are an unbiased approach to test for associations between a specific genetic variant and a wide range of phenotypes in large numbers of individuals ${ }^{7,15}$. By exploring the associations of a genetic variant that impacts the function of a drug target gene, PheWAS in RWD cohorts may enrich the drug discovery 
process for five reasons: 1) association studies in RWD cohorts may validate target-disease links in cohorts that more closely resemble the "real-world", i.e. the patients that will ultimately receive $\operatorname{a~drug}^{16}$; 2) by unraveling pleiotropy, PheWAS may improve our understanding of the biological functions of a target, or hint at concealed pathophysiological connections between disease entities previously considered as distinct ${ }^{17,18}$; 3) PheWAS may reveal opportunities for drug repurposing, an attractive alternative to de novo drug development ${ }^{19,20}$; 4) PheWAS may point to phenotypes that associate with an inverse directionality of target function, thus unravelling potential ADEs at very early stages of a developmental program, minimize risks to trial participants, and help define the most appropriate patient populations to benefit from a drug $\left.{ }^{20} ; 5\right)$ through quantitative estimates from genetic safety and efficacy profiles, PheWAS may help prioritize multiple possible targets by identifying the target with the most promising therapeutic window. Despite these benefits the ability for PheWAS to substantially add to the decision making in drug development is thwarted by the difficulty to obtain and systematize comprehensive genotypes and phenotypes across very large numbers of individuals.

Here, we have tested the hypothesis that PheWAS can inform target validation at early stages of drug development. We selected candidate drug targets across a range of therapeutic indications based on their support from genome-wide association studies (GWAS). To maximize power, we mapped a large spectrum of clinical endpoints from four of the world's largest RWD population cohorts and conducted association testing in up to 697,815 individuals. Our results show that PheWAS, despite limitations, enrich drug discovery with valuable information. 


\section{RESULTS}

\section{Assessing pleiotropy for SNPs in/near 19 drug targets through meta-analyses across four Real-World Data cohorts}

In this study, we queried the literature for genes nominated through GWAS as putatively causally linked to the risk for common complex human diseases and supported by various degrees of additional genetic or biological evidence. We selected 19 genes that, based on previously described genetic associations with either immune-mediated (9 genes: ATG16L1, CARD9, CD226, CDHR3, GPR35, GPR65, IFIH1, IRF5, TYK2), cardiometabolic (8 genes: F11, F12, GDF15, GUCY1A3, KNG1, LGALS3, PNPLA3, SLC30A8) or neurodegenerative diseases (2 genes: LRRK2, TMEM175), were evaluated as potential novel drug targets. Gene-disease associations had been established through 25 common lead single nucleotide polymorphisms (SNPs) that all reached a conservative level of statistical significance $\left(\mathrm{P}<5 \times 10^{-8}\right)$ for association in GWAS with at least one phenotype of relevance to drug discovery and development (Table S1). All of these SNPs have either been demonstrated to impact the target gene in functional studies (genetic evidence), or locate proximal to a gene implicated in a biological mechanism related to the GWAS phenotype (biological evidence). Our selection ranged from targets with little biological knowledge beyond GWAS nomination (e.g. TMEM175 for Parkinson's disease) to targets with drug candidates in early clinical trials (e.g. F11 for thromboembolism). Details on the genetic and biological support for all selected genes and SNPs is provided in Supplementary Information.

To broadly investigate pleiotropic effects of the 25 chosen SNPs in a maximal number of individuals, we interrogated four large RWD cohorts that link genome-wide genotype data from individuals of European ancestry with extensive phenotypic data: the 23andMe Inc. cohort with 
self-reported phenotypes on 671,151 research participants ${ }^{21}$, the interim UK Biobank cohort analyzed by Genomics plc with questionnaire-based health information on 112,337 participants (from the first genetic data release in May 2015) ${ }^{10}$, and two EHR-based cohorts from an adult Finnish cohort (FINRISK; 21,371 participants) ${ }^{22}$ and from a pediatric healthcare population from the Children's Hospital of Philadelphia (CHOP; 12,044 patients) ${ }^{23}$ (Table 1 and Methods). All four cohorts contributed phenotypic data in different formats (medical interviews, self-reports, WHO ICD codes, or ICD9-CM codes) in both shared and distinct phenotype categories (Fig. 1A). Together, the four RWD cohorts allowed association testing for a total of 1,892 binary endpoints. Manual phenotype mapping identified 145 distinct clinical endpoints that could be reliably harmonized across two or more cohorts, enabling meta-analyses in up to 697,815 individuals (Fig. 1B and Table S2). As illustrated in Fig. 1C, these 145 mapped phenotypes represent a broad spectrum of disease categories and, as typically observed in RWD, show significant variability in the case:control ratios, both within and between cohorts.

\section{Association testing in RWD cohorts validates known GWAS signals}

We first evaluated whether association testing in the four RWD cohorts (referred to as 'RWD PheWAS') replicated established results from published GWAS. GWAS had associated the 25 tested SNPs with genome-wide significance to 58 binary disease endpoints. Of these, 30 endpoints were ascertained with adequate power (beta $\geq 0.8)$ to reach $\mathrm{FDR}<0.1\left(\mathrm{P}<3.8 \times 10^{-4}\right)$ in the RWD cohorts. We observed that 21 of the $30(70 \%)$ powered GWAS associations replicated (FDR $<0.1)$ in our RWD PheWAS meta-analysis (Fig. S1 and Table S3). As expected from data obtained in real-world settings, the replication rate of known associations was highly disease-dependent. For instance, out of the 9 associations that failed to replicate despite sufficient case numbers in the 
cohorts, 6 were associations with inflammatory bowel disease (IBD), Crohn's disease (CD) or ulcerative colitis (UC), likely reflecting suboptimal ascertainment of these endpoints in real-world settings. Nonetheless, the high replication rate of previously reported associations demonstrates the power of combining disease-agnostic RWD cohorts from various sources to detect and validate true SNP-disease associations, and to substantiate therapeutic hypotheses.

\section{Meta-PheWAS across RWD cohorts identify novel SNP-endpoint associations}

We next investigated whether meta-PheWAS across the four RWD cohorts could identify novel associations to support the proposed clinical indication, suggest alternative indications for drug repositioning, or uncover potential target-related ADEs. To improve statistical power in this analysis, the RWD meta-PheWAS results were further combined with summary statistics from published GWAS studies of 34 diseases available from a larger database assembled and harmonized by Genomics plc (referred to as Genomics plc GWAS, Supplementary Information). Overall, 27,763 association tests (across 145 harmonized and 1,538 cohort-specific endpoints) resulted in 10 putative novel associations reaching study-wide significance after Bonferroni correction $\left(\mathrm{P}<1.8 \times 10^{-6}\right)$ (Table 2). Using a less stringent significance threshold of FDR $<0.1$ $\left(\mathrm{P}<7 \times 10^{-4}\right)$ previously applied in $\mathrm{PheWAS}^{24}$, we identified 72 distinct putative novel associations

(Fig. 2, Fig. S2, Table S4 and Supplementary Datasheet). Forty-four of these putative novel associations showed directions of effect consistent with the proposed clinical indication for a drug and may hint at potential repositioning opportunities. Conversely, 27 showed directions of effect opposite to the proposed clinical indication and may suggest safety signals that could endanger therapeutic success and warrant monitoring for in preclinical models and clinical trials. 


\section{Apparent pleiotropy and endpoint co-morbidities challenge target validation through}

\section{PheWAS}

A challenge to the PheWAS approach is to reliably distinguish true pleiotropic associations of a SNP (or SNPs in strong LD with the lead SNP), suggesting a shared causal mechanism, from unrelated associations driven by independent SNPs at a locus ${ }^{17}$. For instance, in our metaPheWAS, the putative association of rs2274273 near LGALS3 (encoding the galactin-3 protein) with Parkinson's disease $(\mathrm{PD})\left(\mathrm{OR}=0.94, \mathrm{P}=1 \times 10^{-4}\right)$ likely reflects a distinct causal mechanism previously attributed to $G C H 1^{25}$. rs 2274273 is a protein quantitative trait locus (pQTL) that controls plasma levels of galectin- $3^{26}$. Through a Bayesian test for co-localization using summary statistics from published GWAS studies ${ }^{27,28}$, we excluded rs2274273 as a causal SNP for PD (posterior probability for a shared variant leading the PD and galectin-3 levels associations = 0.0008\%) (Fig. S3).

A second challenge to PheWAS is the existence of common co-morbidities among endpoints, or alternatively an insufficient distinction between phenotypes ${ }^{18}$. In our meta-PheWAS, rs17724992 near GDF15 showed association with multiple cardiovascular-related phenotypes, which is likely mediated by the known association of this SNP with body mass index (BMI) ${ }^{29}$, an established risk factor for cardiovascular disease $\mathrm{e}^{30}$. This is supported by the lack of association of rs 17724992 with coronary artery disease (CAD) in the large GWAS published by the CARDIoGRAMplusC4D consortium $(\mathrm{P}=0.17)^{31}$. Follow-up customized association analyses adjusting for specific phenotypic covariates are required to distinguish true pleiotropic effects and inform target validation.

In summary, these two examples demonstrate that thorough investigation of association results can reduce biases introduced through PheWAS. 


\section{Pleiotropy of rs738409 (p.I148M) predicts a risk for multiple potential ADEs upon inhibiting}

\section{PNPLA3}

Among the 10 study-wide significant associations, our meta-PheWAS revealed multiple novel associations for the PNPLA3 missense SNP rs738409 (p.I148M). The rs738409-G allele has previously been reported as associated with an increased risk for non-alcoholic fatty liver disease (NAFLD), alcohol-related cirrhosis and hepatic steatosis, as well as elevated alanine aminotransferase (ALT) levels, most likely through a gain-of-function (GOF) mechanism (Supplementary Information). Consistent with these findings, our meta-PheWAS found rs 738409-G to be associated with elevated liver tests $\left(\mathrm{OR}=1.25, \mathrm{P}=4 \times 10^{-45}\right)$ (Fig. S4). Beyond that, our analysis also indicated that carriers of the rs $738409-\mathrm{G}$ allele that increases ALT are more prone to develop liver toxicities when treated with nonsteroidal anti-inflammatory drugs (NSAIDs) such as ibuprofen $\left(\mathrm{OR}=1.43, \mathrm{P}=4.6 \times 10^{-5}\right)$ or aspirin $\left(\mathrm{OR}=1.57, \mathrm{P}=5.3 \times 10^{-5}\right)$. In addition, the metaPheWAS revealed significant associations between rs738409-G and an increased risk of T2D $\left(\mathrm{OR}=1.08, \mathrm{P}=8 \times 10^{-11}\right)$, as well as a decreased risk for acne $\left(\mathrm{OR}=0.90, \mathrm{P}=1.5 \times 10^{-11}\right)$, high cholesterol $\left(\mathrm{OR}=0.96, \mathrm{P}=1.6 \times 10^{-7}\right)$ or the intake of cholesterol-lowering medications $(\mathrm{OR}=0.97$, $\left.\mathrm{P}=2 \times 10^{-4}\right)$, gout $\left(\mathrm{OR}=0.92, \mathrm{P}=4.1 \times 10^{-5}\right)$, and gallstones $\left(\mathrm{OR}=0.95, \mathrm{P}=2.7 \times 10^{-4}\right)$. All these associations remained prominent after adjusting for elevated liver tests (Table S5). Associations of rs 738409-G with T2D and high cholesterol were supported by independent recent data from the GoT2D 82k exome chip study $\left(\mathrm{OR}=1.06, \mathrm{P}=7.7 \times 10^{-5}\right)$ and the LDL GLGC $300 \mathrm{~K}$ exome chip study $\left(\right.$ Beta $\left.=-0.018, \mathrm{P}=1 \times 10^{-8}\right)$, where fine-mapping confirmed rs 738409 to be the most likely causal $\mathrm{SNP}^{32,33}$. Taken together, our PheWAS results support the hypothesis that therapeutic inhibition of PNPLA3 could treat liver diseases. They also support T2D as a potential alternative indication 
for PNPLA3 inhibition. However, concomitant inverse associations with multiple other endpoints, including acne and high plasma cholesterol levels, indicate potential clinically relevant on-target ADEs that should be considered in decisions to progress PNPLA3 inhibitors towards clinical development.

\section{IFIH1 partial loss-of-function increases the genetic risk for asthma}

As another example of pleiotropic effects identified in our meta-PheWAS, carriers of the IFIHI (encoding MDA5) rs1990760-C allele (MAF=40\%) have an established lower risk for several autoimmune diseases (type 1 diabetes, T1D; vitiligo; systemic lupus erythematosus, SLE; psoriasis) and an increased risk for ulcerative colitis (UC) (Supplementary Information). Functional studies suggest that rs1990760-C (p.T946A) causes IFIH1 loss-of-function (LOF), and additional IFIH1 LOF alleles have been shown to protect against T1D, vitiligo, psoriasis and psoriatic arthritis (PsA) (Supplementary Information). Our meta-PheWAS support these associations (Fig. 2 and Table S3). Importantly, we also found a significant novel association between rs1990760-C and increased risk for asthma $\left(\mathrm{OR}=1.04, \mathrm{P}_{\text {meta }}=9.0 \times 10^{-8}\right)$ (Fig. 3A). The association between rs1990760 and asthma was supported by data from all four RWD cohorts as well as the GABRIEL and EVE asthma GWAS cohorts $\left(\mathrm{OR}_{\text {meta }}=1.04, \mathrm{P}_{\text {meta }}=6.5 \times 10^{-8}\right)^{34,35}$, despite lack of power to detect an association with rs1990760 in the published GWAS cohorts alone (Fig. 3B). This association remained significant after adjustment for autoimmune diseases in the 23andMe cohort, demonstrating that the asthma association is independent of the previously established associations of rs1990760 with autoimmunity (Table S6). Co-localization analysis confirmed that the same SNP was responsible for the SLE, UC and asthma associations at the locus, supporting true pleiotropic effects driven by the same causal variant(s) (Fig. 3C). The 
observed IFIH1 pleiotropic effects were further strengthened by the observation in the Genomics plc UK biobank data that the independent low-frequency IFIH1 missense allele p.I923V (rs35667974-C, MAF=1.8\%), previously reported to result in IFIH1 LOF and to protect against T1D, vitiligo, psoriasis, and PsA, and to increase risk of UC, was also associated with increased risk of asthma $\left(\mathrm{OR}=1.18, \mathrm{P}=1.1 \times 10^{-4}\right)$, appearing as the top asthma-associated SNP at the locus (Fig. 3D and Fig. S5). Together, these and previous findings establish IFIH1 as a gene with an "allelic series" 36 and further support the therapeutic hypothesis that inhibition of MDA5 may protect against autoimmune disease. However, our results also reveal the potential of MDA5 inhibitors to cause pulmonary ADEs and strengthen previous findings for an increased risk for colitis-related symptoms, endpoints that may limit the therapeutic window of MDA5 modulators and should be considered for monitoring in clinical trials.

\section{Genetic efficacy and safety signals assist target prioritization for thromboembolism}

Beyond informing on individual genes, we hypothesized that PheWAS might help prioritize targets among several candidates within a biological pathway. Factors XI, XII and plasma kininogen (encoded by $K N G 1$ ) are members of the contact activation coagulation pathway ${ }^{37}$. Anticoagulation therapies directed against these factors are hypothesized to have improved therapeutic windows over current standard-of-care, which is accompanied by significant bleeding liabilities ${ }^{38}$. With the aim to estimate genetic risk-benefit profiles for the three candidate targets, we chose to interrogate three uncorrelated SNPs at the F11, KNG1 and F12 loci. These three SNPs had similar allele frequencies in Europeans, had previously been shown to impact FXI, FXII and/or KNG1 mRNA and/or protein levels, and are associated with activated partial thromboplastin time (aPTT), a biomarker of blood clotting, or venous thromboembolism (VTE), risk (Supplementary 
Information and Table S1). Carriers of the rs4253399-T allele, which reduces circulating FXI levels and increases aPTT, showed an expected lower risk for blood clots $\left(\mathrm{OR}=0.84, \mathrm{P}=3.5 \times 10^{-}\right.$ $\left.{ }^{25}\right)$, but no evidence for association with bleeding tendency $(\mathrm{OR}=1.04, \mathrm{P}=0.35)$ (Fig. 4). In contrast, carriers of the KNG1 allele rs5030062-A, which reduces plasma kininogen as well as circulating FXI, and inceases aPTT, showed both reduced blood clotting $\left(\mathrm{OR}=0.93, \mathrm{P}=1.6 \times 10^{-4}\right)$ as well as increased bleeding liability $\left(\mathrm{OR}=1.14, \mathrm{P}=4.1 \times 10^{-4}\right)$. A nominal association with both traits was found in carriers of the FXII levels-reducing and aPTT-increasing allele rs2731672-T (blood clots: $\mathrm{OR}=0.96, \mathrm{P}=0.034$; bleeding tendency: $\mathrm{OR}=1.09, \mathrm{P}=0.039$ ). By comparing these results with the effect of the three SNPs on aPTT (Table S1), our study suggests that, among the three factors tested, targeting FXI may yield the best compromise between thromboembolism risk reduction and increased bleeding liability, which is consistent with the outcomes of a recent phase 2 clinical trial $^{39}$. 


\section{DISCUSSION}

Our study investigates the utility of PheWAS for predicting therapeutic success of candidate drug targets nominated through human genetics. We focused on a selection of loci that GWAS have firmly established as associated with common immune-mediated, cardio-metabolic, or neurodegenerative human diseases, and where additional biological or genetic evidence supports candidate drug target genes within these loci as likely causing the disease associations. We analyzed SNPs impacting these targets for association with 1,683 disease endpoints captured in four large disease-agnostic "real-world" population cohorts that link genome-wide genotypes with various types of structured health information. Our PheWAS meta-analysis replicates $70 \%$ of the published GWAS associations at FDR $<0.1$, substantially surpassing performance of previous PheWAS in smaller cohorts ${ }^{24}$. Through joining PheWAS results with published GWAS data, we identified 10 novel SNP-phenotype associations that exceeded stringent significance thresholds for multiple test correction, as well as additional putative associations with therapeutically relevant clinical endpoints. For a subset of prominent early drug targets, our results support previous genetic evidence for efficacy in distinct common disease indications. Our analysis further proposes alternative indications as opportunities for drug repositioning, and predicts on-target adverse drug events that may warrant preclinical or clinical monitoring.

Among others, we discovered novel associations for p.I148M in PNPLA3. This is a common gainof-function missense allele increasing the risk for a range of liver phenotypes, which suggested that pharmaceutical inhibition of PNPLA3 could be a viable strategy to treat or prevent liver diseases. While our PheWAS support this hypothesis and further expand the indication spectrum of a putative PNPLA3 inhibitor to T2D, they also uncovered associations with severe acne and 
high cholesterol, phenotypes that if observed only during a clinical trial might put a therapeutic program at risk.

We also identified a novel association of the IFIH1 loss-of-function allele rs1990760-C (p.T946A) with risk of asthma. The rs1990760-C allele, which protects against autoimmune diseases and increases risk of ulcerative colitis, has been shown to decrease interferon (IFN) signaling and lower resistance to viral challenge ${ }^{40}$, while complete loss-of IFIH1 function makes children susceptible to severe viral respiratory infections ${ }^{41,42}$. The association of $\mathrm{rs} 1990760-\mathrm{C}$ with increased risk of asthma discovered in our meta-PheWAS is consistent with the observation that bronchial epithelial cells from asthmatics produce lower amounts of IFN- $\beta$ during viral infections ${ }^{43}$, a finding that lead to inhaled IFN- $\beta$ being tested in phase 2 clinical trials for the treatment of virus-induced asthma exacerbation $^{44}$. Future studies will need to investigate the risk:benefit ratio of modulating MDA5 (encoded by $I F I H 1$ ) for asthma relative to autoimmune disease.

While our study illustrates the power of systematically interrogating RWD cohorts to enrich target validation, it also emphasizes several opportunities to improve existing resources in order for PheWAS to become a routine tool in drug discovery and development. First, truly large, thoroughly phenotyped cohorts will be needed to adequately power PheWAS. Despite our study being conducted in more than 800,000 individuals, about one third of GWAS associations could not be replicated in the RWD cohorts at a stringent level of statistical significance due to an insufficient number of cases. In addition, PheWAS should considerably gain from improved ascertainment of phenotypes ${ }^{45}$. In our study, this is best reflected by an only modest replication rate, despite adequate power, for $\mathrm{CD}, \mathrm{UC}$ and $\mathrm{IBD}$ endpoints that are closely related and difficult 
to discern in routine clinical settings. To better take these considerations and other characteristics of RWD cohorts (typical case:control ratio unbalance between phenotypes, and phenotype correlation) into account, novel statistical methods will be needed to better define significance thresholds and control type I error rates in $\mathrm{PheWAS}^{46}$. Second, our study highlights the challenge to systematically combine phenotypes from independent RWD cohorts. While we introduce the concept of "meta-PheWAS" and demonstrate that mapping phenotypes to interrogate independent PheWAS cohorts may considerably strengthen association signals, standardized terminology, automated phenotype extraction, and coordinated data management across healthcare institutions such as within the eMERGE network should help with better harmonization across cohorts in the future $^{9,47}$. A third challenge to the PheWAS approach is inherent to the current limitations of human genetics. Even when starting from a highly-annotated set of loci as in our study, PheWAS may lead to spurious associations that can only be ruled out through thorough follow-up ${ }^{17}$. We demonstrate this at the example of $L G A L S 3$ and Parkinson's disease. Access to genome-wide association results for systematic fine-mapping and co-localization analyses, functionalization of GWAS loci and the emergence of association data for intermediate phenotypes, e.g. at the protein level, will be needed to help narrow the gap between SNPs and candidate target genes in the future. Finally, a fourth challenge to broadly use PheWAS for drug development is to relate findings from germline variants that impact a target across an individual's entire lifetime to success of an interventional trial with much shorter observation periods. In the end, many decisions to pursue or discontinue a therapeutic program may remain dependent on the specific risk/benefit ratio that quantitative genetics as applied here may help to predict, and the level of unmet clinical need. 
Taken together, our study highlights PheWAS as a highly promising, yet largely untapped opportunity to use disease-agnostic "real world" cohorts for drug target validation. We provide several examples that illustrate PheWAS as a powerful strategy to help predict efficacy and unintended drug effects, which should ultimately help to develop better drugs. Whether PheWAS may truly impact decision-making during drug development will only become evident with either the emergence of ADEs in trials that genetics could have predicted, or reduced safety-related attrition rates for portfolios enriched in targets nominated through human genetics. The growing number of large-scale population cohorts that link genetic with extensive clinical data, together with an increased willingness across the borders of academia, biotech and the pharmaceutical industry to collaborate and share data, will provide opportunities to demonstrate that. 


\section{MATERIALS AND METHODS}

\section{SNP selection}

In this study, we selected 25 SNPs that were significantly associated $\left(\mathrm{P}<5 \times 10^{-8}\right)$ in published GWAS with binary or quantitative phenotypes related to three main therapeutic areas: (auto)immune, cardiometabolic, or neurodegenerative diseases (Supplementary Information).

These 25 SNPs had either been functionally validated in published studies, establishing the candidate target gene as causal for the risk of disease, or they were located within or near genes for which previous studies had generated convincing biological evidence to be of relevance for the respective clinical endpoint. The 25 SNPs were linked to 19 genes that were evaluated as candidate drug targets. Detailed information on the SNPs, candidate causal genes and their link to common human disease is provided in Supplementary Information. The list of SNPs and their known associated phenotypes is provided in Table S1.

\section{Study cohorts}

We interrogated four large observational disease-agnostic RWD cohorts of subjects of European ancestry with genome-wide genotyped data linked to extensive phenotypic information (Table 1). All participants included in each of the four cohorts were unrelated individuals of European ancestry. Individual-level data from each cohort was analyzed independently, and the relevant summary statistics for the 25 SNPs were shared for further analysis. We restricted all cohorts to binary disease phenotypes with at least 20 cases per cohort.

1) 23 andMe: The 23 andMe cohort comprised up to 671,151 participants and 654 binary disease endpoints derived from questionnaire-based self-reports ${ }^{21}$. Participants were restricted to a set of 
individuals who have $>97 \%$ European ancestry, as determined through an analysis of local ancestry using a support vector machine (SVM) and a hidden Markov model (HMM) to assign individuals to one of 31 reference populations. A maximal set of unrelated individuals was chosen for each phenotype using a segmental identity-by-descent (IBD) estimation algorithm. Individuals were defined as related if they shared more than $700 \mathrm{cM}$ IBD, including regions where the two individuals share either one or both genomic segments identical-by-descent. SNPs with HardyWeinberg equilibrium $P<10^{-20}$, call rate $<95 \%$, or with large allele frequency discrepancies compared to European 1000 Genomes reference data were excluded. Participant genotype data were then imputed against the September 2013 release of 1000 Genomes Phase1 reference haplotypes ${ }^{48}$, using an internally developed phasing tool, Finch, which implements the Beagle haplotype graph-based phasing algorithm ${ }^{49}$, and Minimac $2^{50}$.

2) Genomics plc UK Biobank: The Genomics plc analysis of UK biobank cohort (referred to as 'Genomics plc UK Biobank') comprised 112,337 participants and 90 binary disease endpoints derived from questionnaire-based self-reports and medical interviews ${ }^{10}$. GWAS analyses were performed by Genomics plc using the interim data release (May 2015). QC followed the recommendations provided by UK Biobank. European ethnicity was defined as self-reported "white British" ethnic background, and confirmed by principal component analysis clustering. Samples with relatives (3rd degree or closer) were excluded. Imputation was carried out by the UK Biobank data providers using SHAPEIT3 ${ }^{51}$, IMPUTE3 ${ }^{52}$, and a reference panel combining the 1000 Genomes Phase $3^{53}$ and UK10K datasets ${ }^{54}$.

3) FINRISK: FINRISK is a collection of cross-sectional population surveys carried out since 1972 to assess the risk factors of chronic diseases and health behavior in the working age population of Finland $^{22}$. The FINRISK cohort comprised 21,371 Finnish participants and 269 binary disease 
endpoints derived from ICD codes grouping in Finnish national hospital registries and cause-ofdeath registry, and drug reimbursement and purchase registries. The FINRISK samples were genotyped using Illumina CoreExome, OMNIExpress, and $610 \mathrm{~K}$ chips. After gender check, samples with genotype missing rate $>5 \%$ or excess herozygosity $(>4 \mathrm{SD})$ were excluded. SNPs QC, including exclusion of SNPs with genotype missing rate $>2 \%$, minor allele frequency $<1 \%$, or Hardy-Weinberg equilibrium $\mathrm{P}$ value $<1 \times 10^{-6}$, was performed for each genotyping chip separately. Multidimensional scaling (MDS) components were estimated with PLINK v1.9 $9^{55}$ from the LD-pruned genotype data where relatives with pi-hat $>0.2$ had been removed. Samples with non-Finnish ancestry observed as MDS outliers were removed. Imputation was performed with SHAPEIT $^{51}$ and IMPUTE2 ${ }^{52}$ using a reference panel combining information from the 1000 Genomes phase $3^{53}$ and 1,941 Finnish SiSu whole genome sequences ${ }^{56}$. Imputation was stratified based on genotyping chip.

4) CHOP: The cohort from the Children's hospital of Philadelphia (CHOP) comprised 12,044 pediatric patients and 870 binary disease endpoints derived from ICD9-CM codes using the ICD9to-PheWAS codes mapping described by Denny et $a l^{23,57}$. Subjects included in the CHOP PheWAS were genotyped on one of the following genotyping chips following the Illumina standard protocols: Illumina Human610-Quad version 1, Illumina 550K SNP array, or Illumina OmniExpress array. Samples with genotype call rate above 95\% were included in the study. SNPs with genotype missing rate $>5 \%$, minor allele frequency $<1 \%$, and Hardy-Weinberg equilibrium $\mathrm{P}$ value $<0.00001$ were excluded. Principle component analysis (PCA) was performed using EIGENSTRAT $^{58}$ on approximately 130,000 SNPs that had been pruned for linkage disequilibrium using PLINK v1.07 55 and reference genotypes from the HapMap consortium ${ }^{59}$. Imputation was 
performed with SHAPEIT v $2^{51}$ and IMPUTE2 $2^{52}$ using the 1000 Genomes project phase 1 reference panel $^{48}$. SNPs with with INFO scores $<0.9$ were excluded.

All the participants in the 23andMe, Genomics plc UK Biobank, FINRISK and CHOP cohorts provided written informed consent for participating in research studies. Blood samples were collected according to protocols approved by local institutional review boards. This research has been conducted using the UK Biobank resource under the Genomics plc project application number 9659.

In addition, with the aim to replicate novel associations identified in the RWD cohorts, we interrogated genome-wide summary statistics from 57 published GWAS, including 34 binary disease phenotypes, derived from a larger database that has been assembled and harmonized by Genomics plc (referred to as 'Genomics plc GWAS'). The full list of studies in Genomics plc GWAS database and tested in this study is available in the Supplementary Information). These included checks to ensure consistency of the data, and alignment of alleles to the forward strand of the human reference sequence, with effects ascribed to the alternative allele. Effect size estimates for quantitative traits were rescaled relative to the residual variance. Summary-statistic imputation was applied to infer association evidence at common variants (minor allele frequency $>2 \%$ ) in the 1000 Genomes EUR reference panel. Results for SNPs associated with the relevant phenotype with $\mathrm{P}<0.05$ were included in the meta-analysis.

Correlation between all GWAS was estimated to ensure that no GWAS included in the metaanalysis for a given phenotype presented overlapping samples. In addition, to further prevent GWAS results from overlapping samples to be meta-analyzed, only the most recent/largest study for a given disease was included in our analysis when several GWAS studies in the Genomics plc database investigated the same disease. Although we could not directly estimate potential 
overlapping samples between the different RWD cohorts, significant overlap is very unlikely based on the participants' characteristics (Table 1).

\section{Identification of shared phenotypes}

The phenotypic endpoints tested in the different RWD cohorts were derived from different sources (self-reports, medical interviews, WHO ICD codes, ICD9-CM codes) and named with different coding systems (e.g. clinical terms versus popular terms, abbreviations versus full names). In order to compare and combine results from the four RWD cohorts with published GWAS results from the Genomics plc database, we manually mapped the phenotypes. This step allowed us to identify 145 distinct phenotypes shared by at least 2 cohorts and with at least 20 cases in the independent cohorts (Fig. 1). The full list of mapped phenotypes is provided in Table S2. We note that, in each cohort some phenotypes were captured multiple times by different endpoints with slightly different definitions. In this case, only one endpoint per cohort was selected for meta-analysis.

\section{PheWAS and meta-analysis}

Phenome-wide association analyses for each of the 25 SNPs were conducted in the 23andMe, Genomics plc UK biobank, FINRISK (PheWAS results release November 2016) and CHOP cohorts separately. Each SNP-phenotype association was tested independently (assuming an additive genetic model), using logistic regressions adjusted for age, gender, and principal components to adjust for population stratification. Genotyping batch and survey cohort were also included as covariates in the FINRISK PheWAS. We then performed two distinct analyses to 1) replicate known GWAS associations, and 2) to detect novel associations. 
First, we meta-analyzed PheWAS results from the 4 RWD cohorts, to investigate the ability of these cohorts to replicate known GWAS associations. After harmonizing the effect alleles across the cohorts, fixed effect and random effect meta-analyses were performed using PLINK ${ }^{55}$. We then compared the meta-analysis association results with known significant SNP-phenotype associations from published GWAS, taking into account the statistical power to detect an association in the meta-analysis of the PheWAS results in the disease-agnostic RWD cohorts.

Second, we meta-analyzed results from the four disease-agnostic cohorts together with available GWAS results in order to detect novel associations. Meta-analysis was performed using PLINK as described above. Meta-analysis results at the 145 shared phenotypes were then combined with cohort-specific phenotype results from the 25 SNPs, resulting in 27,762 tests in total. False discovery rate (FDR) was calculated for the 27,762 tests combined using the Benjamini and Hochberg method using the $\mathrm{R}$ p.adjust method ${ }^{60}$. The threshold for significance in detecting putative novel associations was established using an FDR of 0.1 , corresponding to $\mathrm{P}<7 \times 10^{-4}$. Significance threshold based on Bonferroni correction was $\mathrm{P}=0.05 / 27,762=1.8 \times 10^{-6}$. We note that Bonferroni correction ignores the correlation structure between the tested phenotypes or the fact that all the SNPs tested in this study are known to be associated with one or several phenotypes in published GWAS.

\section{Statistical power estimations}

We estimated statistical power to detect an association with known associated phenotypes, based on the published effect size in the most recently published GWAS, the frequency of the associated SNP risk allele in the 1000 Genomes EUR population, the number of cases and controls in the disease-agnostic cohorts, and assuming a phenotype prevalence of $1 \%{ }^{61}$. 


\section{Co-localization analyses}

To distinguish true pleiotropic effects from multiple associations at the loci that are explained by different causal SNPs (and potentially incriminating different causal genes), we used association summary statistics available from published GWAS and applied a Bayesian test implemented in the R package 'coloc' to assess co-localization, i.e. the probability of sharing causal genetic variants between pairs of apparent pleiotropic phenotypes using association summary statistics at the loci of interest $^{27}$. Co-localization analysis at the LGALS3 locus was performed using meta-analyzed PD GWAS summary statistics from 23andMe published elsewhere ( $\mathrm{N}$ cases=4,127, $\mathrm{N}$ controls $=62,037)^{25}$, and galectin-3 plasma pQTL results in 3,562 blood donors ${ }^{33}$. Co-localization analysis at the IFIH1 locus was performed using meta-analyzed SLE GWAS results from two independent published studies ${ }^{62,63}$, meta-analyzed asthma GWAS summary statistics from 23 andMe ${ }^{64}$ and the Genomics plc UK Biobank (unpublished), and published UC GWAS summary statistics $^{65}$. 


\section{References:}

1. Plenge RM. Disciplined approach to drug discovery and early development. Sci Transl Med 8, 349ps315. doi: 310.1126/scitranslmed.aaf2608. (2016).

2. Waring MJ, et al. An analysis of the attrition of drug candidates from four major pharmaceutical companies. Nat Rev Drug Discov 14, 475-486. doi: 410.1038/nrd4609. Epub 2015 Jun 1019. (2015).

3. Cook D, et al. Lessons learned from the fate of AstraZeneca's drug pipeline: a fivedimensional framework. Nat Rev Drug Discov 13, 419-431. doi: 410.1038/nrd4309. Epub 2014 May 1016. (2014).

4. Nelson MR, et al. The support of human genetic evidence for approved drug indications. Nat Genet 47, 856-860. doi: 810.1038/ng.3314. Epub 2015 Jun 1029. (2015).

5. Kohane IS. Using electronic health records to drive discovery in disease genomics. Nat Rev Genet 12, 417-428. doi: 410.1038/nrg2999. Epub 2011 May 1018. (2011).

6. Jensen PB, Jensen LJ, Brunak S. Mining electronic health records: towards better research applications and clinical care. Nat Rev Genet 13, 395-405. doi: 310.1038/nrg3208. (2012).

7. Bush WS, Oetjens MT, Crawford DC. Unravelling the human genome-phenome relationship using phenome-wide association studies. Nat Rev Genet 17, 129-145 (2016).

8. Wei WQ, Denny JC. Extracting research-quality phenotypes from electronic health records to support precision medicine. Genome Med 7, 41. doi: 10.1186/s13073-13015-10166-y. eCollection 12015. (2015).

9. Crawford DC, et al. eMERGEing progress in genomics-the first seven years. Front Genet 5:184., 10.3389/fgene.2014.00184. eCollection 02014. (2014).

10. Sudlow C, et al. UK biobank: an open access resource for identifying the causes of a wide range of complex diseases of middle and old age. PLoS Med 12, e1001779. doi: 1001710.1001371/journal.pmed.1001779. eCollection 1002015 Mar. (2015).

11. Gaziano JM, et al. Million Veteran Program: A mega-biobank to study genetic influences on health and disease. $J$ Clin Epidemiol 70:214-23., 10.1016/j.jclinepi.2015.1009.1016. Epub 2015 Oct 1019. (2016).

12. Dewey FE, et al. Distribution and clinical impact of functional variants in 50,726 wholeexome sequences from the DiscovEHR study. Science 354(6319). aaf6814. doi: 6810.1126/science.aaf6814. (2016). 
13. Leitsalu L, et al. Cohort Profile: Estonian Biobank of the Estonian Genome Center, University of Tartu. Int J Epidemiol 44, 1137-1147. doi: 1110.1093/ije/dyt1268. Epub 2014 Feb 1111. (2015).

14. Ehrenstein V, Nielsen H, Pedersen AB, Johnsen SP, Pedersen L. Clinical epidemiology in the era of big data: new opportunities, familiar challenges. Clin Epidemiol 9:245-250., 10.2147/CLEP.S129779. eCollection 122017. (2017).

15. Hebbring SJ. The challenges, advantages and future of phenome-wide association studies. Immunology 141, 157-165. doi: 110.1111/imm.12195. (2014).

16. Denny JC, Bastarache L, Roden DM. Phenome-Wide Association Studies as a Tool to Advance Precision Medicine. Annu Rev Genomics Hum Genet 17:353-73., 10.1146/annurev-genom-090314-024956. Epub 092016 May 090314. (2016).

17. Solovieff N, Cotsapas C, Lee PH, Purcell SM, Smoller JW. Pleiotropy in complex traits: challenges and strategies. Nat Rev Genet 14, 483-495. doi: 410.1038/nrg3461. Epub 2013 Jun 1011. (2013).

18. $\mathrm{Hu}$ JX, Thomas CE, Brunak S. Network biology concepts in complex disease comorbidities. Nat Rev Genet 17, 615-629. doi: 610.1038/nrg.2016.1087. Epub 2016 Aug 1038. (2016).

19. Rastegar-Mojarad M, Ye Z, Kolesar JM, Hebbring SJ, Lin SM. Opportunities for drug repositioning from phenome-wide association studies. Nat Biotechnol 33, 342-345. doi: 310.1038/nbt.3183. (2015).

20. Pulley JM, et al. Accelerating Precision Drug Development and Drug Repurposing by Leveraging Human Genetics. Assay Drug Dev Technol 15, 113-119. doi: 110.1089/adt.2016.1772. Epub 2017 Apr 1085. (2017).

21. Eriksson N, et al. Web-based, participant-driven studies yield novel genetic associations for common traits. PLoS Genet 6, e1000993. doi: 1000910.1001371/journal.pgen.1000993. (2010).

22. Borodulin K, et al. Forty-year trends in cardiovascular risk factors in Finland. Eur J Public Health 25, 539-546. doi: 510.1093/eurpub/cku1174. Epub 2014 Nov 1024. (2015).

23. Grant SF, et al. A genome-wide association study identifies a locus for nonsyndromic cleft lip with or without cleft palate on 8q24. $J$ Pediatr 155, 909-913. doi: 910.1016/j.jpeds.2009.1006.1020. Epub 2009 Aug 1014. (2009).

24. Denny JC, et al. Systematic comparison of phenome-wide association study of electronic medical record data and genome-wide association study data. Nat Biotechnol 31, 11021110. (2013). 
25. Nalls MA, et al. Large-scale meta-analysis of genome-wide association data identifies six new risk loci for Parkinson's disease. Nat Genet 46, 989-993 (2014).

26. de Boer RA, et al. A genome-wide association study of circulating galectin-3. PLoS One 7, e47385. doi: 47310.41371/journal.pone.0047385. Epub 0042012 Oct 0047389. (2012).

27. Giambartolomei C, et al. Bayesian test for colocalisation between pairs of genetic association studies using summary statistics. PLoS Genet 10, e1004383 (2014).

28. Sun BB, et al. Consequences Of Natural Perturbations In The Human Plasma Proteome. bioRxiv, (2017).

29. Locke AE, et al. Genetic studies of body mass index yield new insights for obesity biology. Nature 518, 197-206 (2015).

30. Labounty TM, et al. Body mass index and the prevalence, severity, and risk of coronary artery disease: an international multicentre study of 13,874 patients. Eur Heart J Cardiovasc Imaging 14, 456-463. doi: 410.1093/ehjci/jes1179. Epub 2012 Aug 1024. (2013).

31. Nikpay M, et al. A comprehensive 1,000 Genomes-based genome-wide association metaanalysis of coronary artery disease. Nat Genet 47, 1121-1130. doi: 1110.1038/ng.3396. Epub 2015 Sep 1127. (2015).

32. AMP-T2D Program. PNPLA3. type2diabetesgenetics.org (Apr 15, 2017).

33. Mahajan A, Morris AP, Rotter JI, McCarthy MI. Refining The Accuracy Of Validated Target Identification Through Coding Variant Fine-Mapping In Type 2 Diabetes. bioRxiv, (2017).

34. Moffatt MF, et al. A large-scale, consortium-based genomewide association study of asthma. N Engl J Med 363, 1211-1221. doi: 1210.1056/NEJMoa0906312. (2010).

35. Myers RA, et al. Further replication studies of the EVE Consortium meta-analysis identifies 2 asthma risk loci in European Americans. J Allergy Clin Immunol 130, 12941301. doi: 1210.1016/j.jaci.2012.1207.1054. Epub 2012 Oct 1294. (2012).

36. Plenge RM, Scolnick EM, Altshuler D. Validating therapeutic targets through human genetics. Nat Rev Drug Discov 12, 581-594. doi: 510.1038/nrd4051. Epub 2013 Jul 1019. (2013).

37. Wu Y. Contact pathway of coagulation and inflammation. Thromb $J$ 13:17., 10.1186/s12959-12015-10048-y. eCollection 12015. (2015).

38. Ruff CT, et al. North American Thrombosis Forum, AF Action Initiative Consensus Document. Am J Med 129, S1-S29. doi: 10.1016/j.amjmed.2016.1002.1001. (2016). 
39. Buller HR, et al. Factor XI antisense oligonucleotide for prevention of venous thrombosis. $N$ Engl J Med 372, 232-240. doi: 210.1056/NEJMoa1405760. Epub 1402014 Dec 1405767. (2015).

40. Gorman JA, et al. The A946T variant of the RNA sensor IFIH1 mediates an interferon program that limits viral infection but increases the risk for autoimmunity. Nat Immunol 18, 744-752. doi: 710.1038/ni.3766. Epub 2017 May 1029. (2017).

41. Asgari S, et al. Severe viral respiratory infections in children with IFIH1 loss-of-function mutations. Proc Natl Acad Sci U S A 17, 1704259114 (2017).

42. Lamborn IT, et al. Recurrent rhinovirus infections in a child with inherited MDA5 deficiency. J Exp Med 214, 1949-1972. doi: 1910.1084/jem.20161759. Epub 20162017 Jun 20161712. (2017).

43. Wark PA, et al. Asthmatic bronchial epithelial cells have a deficient innate immune response to infection with rhinovirus. J Exp Med 201, 937-947. (2005).

44. Djukanovic R, et al. The effect of inhaled IFN-beta on worsening of asthma symptoms caused by viral infections. A randomized trial. Am J Respir Crit Care Med 190, 145-154. doi: 110.1164/rccm.201312-202235OC. (2014).

45. Chen $\mathrm{Y}$, et al. Building bridges across electronic health record systems through inferred phenotypic topics. J Biomed Inform 55:82-93., 10.1016/j.jbi.2015.1003.1011. Epub 2015 Apr 1011. (2015).

46. Dey R, Schmidt EM, Abecasis GR, Lee S. A Fast and Accurate Algorithm to Test for Binary Phenotypes and Its Application to PheWAS. Am J Hum Genet 2, 014 (2017).

47. Kho AN, et al. Electronic medical records for genetic research: results of the eMERGE consortium. Sci Transl Med 3, 79re71. doi: 10.1126/scitranslmed.3001807. (2011).

48. Abecasis GR, et al. An integrated map of genetic variation from 1,092 human genomes. Nature 491, 56-65. doi: 10.1038/nature11632. (2012).

49. Browning SR, Browning BL. Rapid and accurate haplotype phasing and missing-data inference for whole-genome association studies by use of localized haplotype clustering. Am J Hum Genet 81, 1084-1097. Epub 2007 Sep 1021. (2007).

50. Fuchsberger C, Abecasis GR, Hinds DA. minimac2: faster genotype imputation. Bioinformatics 31, 782-784. doi: 710.1093/bioinformatics/btu1704. Epub 2014 Oct 1022. (2015).

51. Delaneau O, Zagury JF, Marchini J. Improved whole-chromosome phasing for disease and population genetic studies. Nat Methods 10, 5-6. doi: 10.1038/nmeth.2307. (2013). 
52. Howie BN, Donnelly P, Marchini J. A flexible and accurate genotype imputation method for the next generation of genome-wide association studies. PLoS Genet 5, e1000529. doi: 1000510.1001371/journal.pgen.1000529. Epub 1002009 Jun 1000519. (2009).

53. Sudmant PH, et al. An integrated map of structural variation in 2,504 human genomes. Nature 526, 75-81. doi: 10.1038/nature15394. (2015).

54. Kaye J, et al. Managing clinically significant findings in research: the UK10K example. Eur J Hum Genet 22, 1100-1104. doi: 1110.1038/ejhg.2013.1290. Epub 2014 Jan 1115. (2014).

55. Purcell S, et al. PLINK: a tool set for whole-genome association and population-based linkage analyses. Am J Hum Genet 81, 559-575. Epub 2007 Jul 2025. (2007).

56. Lim ET, et al. Distribution and medical impact of loss-of-function variants in the Finnish founder population. PLoS Genet 10, e1004494. doi: 1004410.1001371/journal.pgen.1004494. eCollection 1002014 Jul. (2014).

57. Denny JC, et al. PheWAS: demonstrating the feasibility of a phenome-wide scan to discover gene-disease associations. Bioinformatics 26, 1205-1210. doi: 1210.1093/bioinformatics/btq1126. Epub 2010 Mar 1224. (2010).

58. Price AL, Patterson NJ, Plenge RM, Weinblatt ME, Shadick NA, Reich D. Principal components analysis corrects for stratification in genome-wide association studies. Nat Genet 38, 904-909. Epub 2006 Jul 2023. (2006).

59. Altshuler DM, et al. Integrating common and rare genetic variation in diverse human populations. Nature 467, 52-58. doi: 10.1038/nature09298. (2010).

60. Benjamini Y, Hochberg Y. Controlling the False Discovery Rate: A Practical and Powerful Approach to Multiple Testing. ( $\mathrm{ed}^{\wedge}(\mathrm{eds})$. Journal of the Royal Statistical Society. Series B (Methodological) (1995).

61. Yang J, Wray NR, Visscher PM. Comparing apples and oranges: equating the power of case-control and quantitative trait association studies. Genet Epidemiol 34, 254-257. doi: 210.1002/gepi.20456. (2010).

62. Bentham J, et al. Genetic association analyses implicate aberrant regulation of innate and adaptive immunity genes in the pathogenesis of systemic lupus erythematosus. Nat Genet 47, 1457-1464 (2015).

63. Hom G, et al. Association of systemic lupus erythematosus with C8orf13-BLK and ITGAM-ITGAX. $N$ Engl $J$ Med 358, 900-909. doi: 910.1056/NEJMoa0707865. Epub 0702008 Jan 0707820. (2008). 
64. Pickrell JK, Berisa T, Liu JZ, Segurel L, Tung JY, Hinds DA. Detection and interpretation of shared genetic influences on 42 human traits. Nat Genet 48, 709-717. doi: 710.1038/ng.3570. Epub 2016 May 1016. (2016).

65. Liu JZ, et al. Association analyses identify 38 susceptibility loci for inflammatory bowel disease and highlight shared genetic risk across populations. Nat Genet 47, 979-986. doi: 910.1038/ng.3359. Epub 2015 Jul 1020. (2015). 


\section{Acknowledgements:}

We thank the research participants from the 23andMe, UK Biobank, FINRISK and CHOP cohorts for their contributions to this study. We would like to thank Jyoti Shah, Jennifer Pai, Mark Sharp, Hongjie Sun and Ian Wallace for their input on phenotype mapping.

\section{Author contributions:}

DD, HR, DFR, JM, JCM, AKC, AB, AD participated in the design and/or interpretation of the reported experiments or results. DD and HR drafted the manuscript. DD performed the phenotype harmonization, meta-analysis and follow-up analyses. RMP and CSF provided supervisory support. CT, the 23andMe Research team and DH participated in the acquisition and/or analysis of the 23andMe data. CSF, CCAS, CV, MEW and PD participated in the acquisition and/or analysis of the Genomics plc UK Biobank data. MA, HM, EK, MR, JH, NB, SJ, DGM, VS, SR, MJD, and AP participated in the acquisition and/or analysis of the FINRISK data. MM, PMAS and HH participated in the acquisition and/or analysis of the CHOP data. 


\section{Figures:}

A

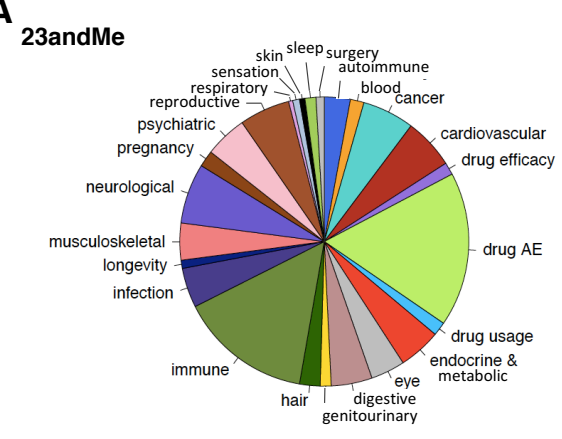

Genomics plc UK Biobank

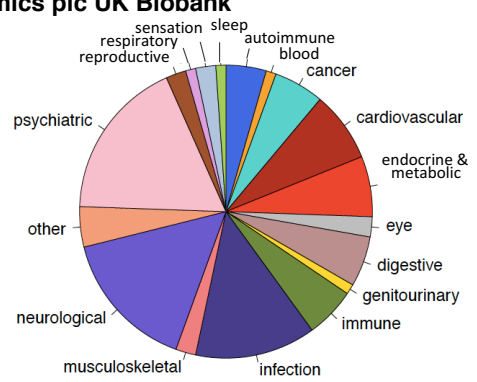

FINRISK

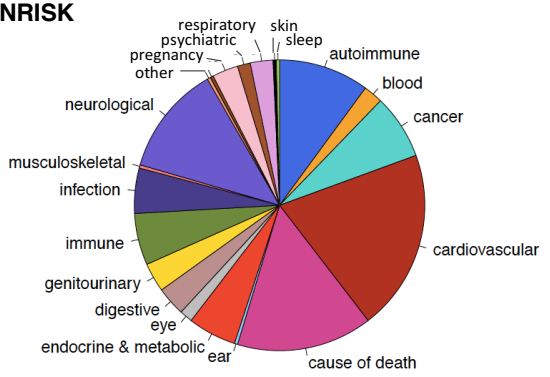

CHOP

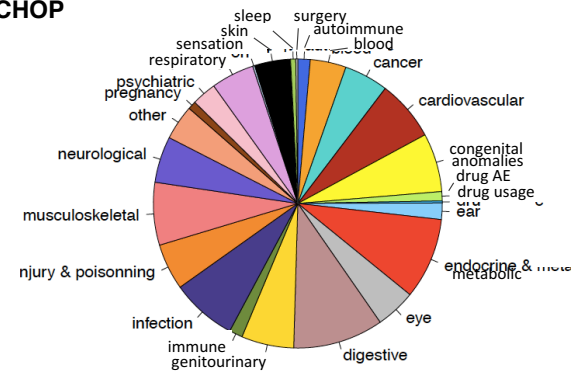

B
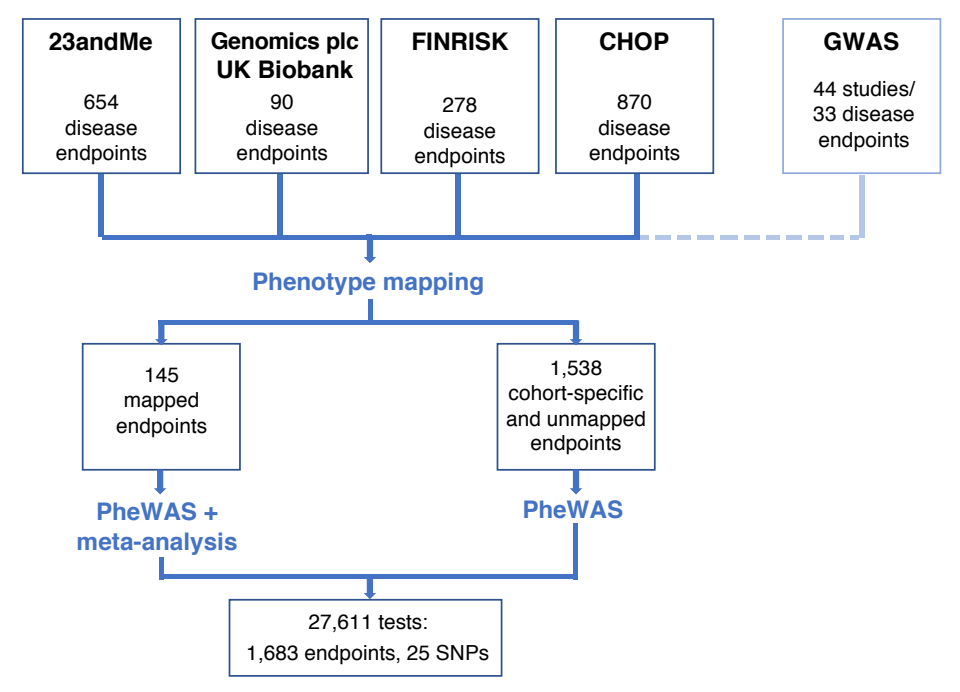

C

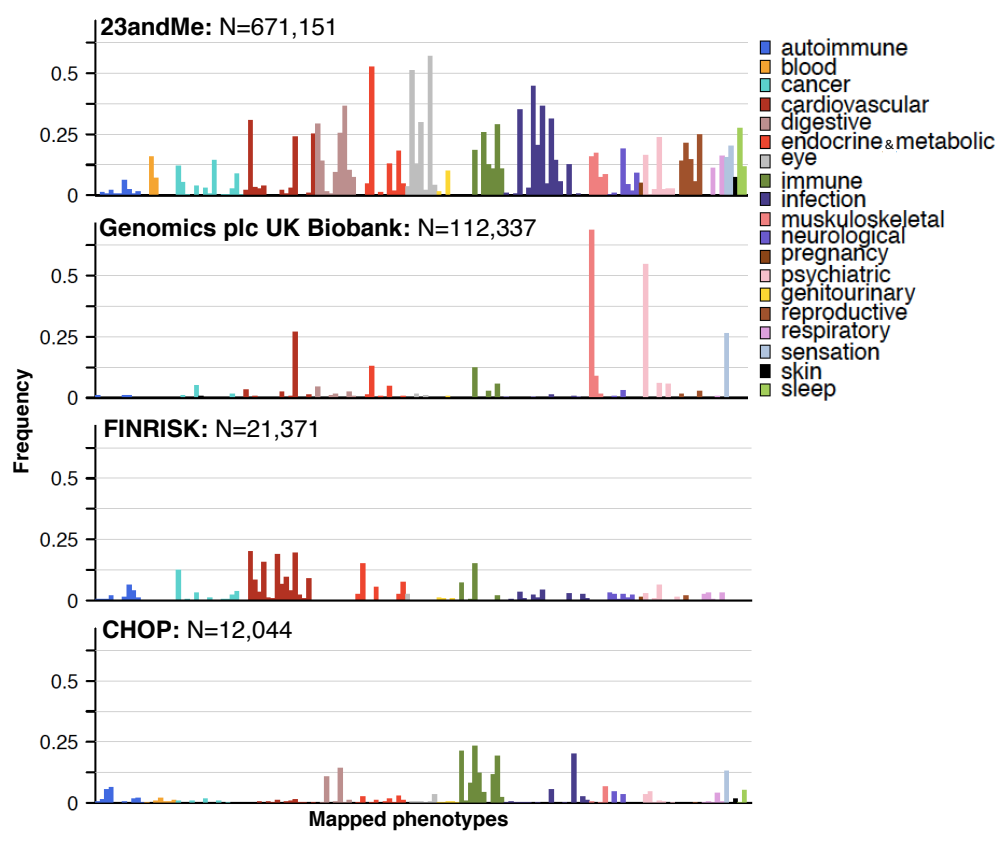

Fig. 1. Phenotypes tested and study design. (A) Categories of phenotypes assessed in the 23andMe, Genomics plc UK Biobank, FINRISK and CHOP RWD cohorts. (B) Manual phenotype mapping was performed to identify phenotypes shared between cohorts. One hundred and forty-five phenotypes were captured with at least 20 cases in at least 2 cohorts. After PheWAS in each cohort separately, the 145 phenotypes were meta-analyzed to increase statistical power and enable systematic comparisons of results between cohorts. (C) The 145 mapped phenotypes (see Supplementary Table 2) represent a broad spectrum of phenotypic categories and are captured with variable case:control ratios in the cohorts tested. 

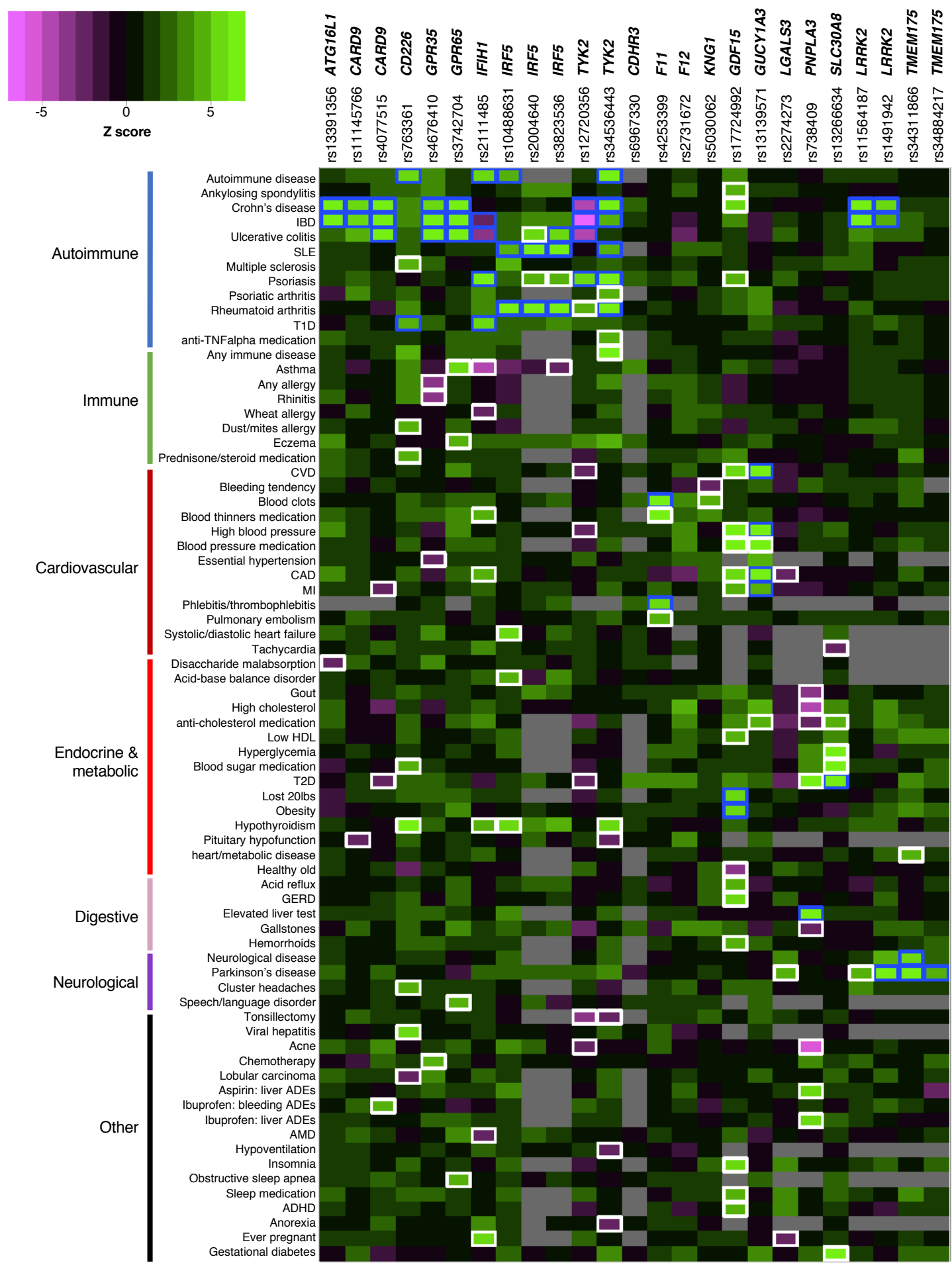

Fig. 2. PheWAS results for 25 GWAS SNPs in/near candidate drug targets from meta-analysis of 4 RWD cohorts with published GWAS data. Phenotypes associated at FDR $<0.1(\mathrm{P}<7 \mathrm{e}-4)$ with at least one SNP in the meta-PheWAS are represented. Direction of effect of the known disease-risk increasing allele related to the therapeutic hypothesis is indicated. A positive Z-score (in green) indicates increased risk, a negative Z-score (in purple) indicates reduced risk. Known and novel associations reaching FDR $<0.1$ are outlined in blue and white respectively. 
A

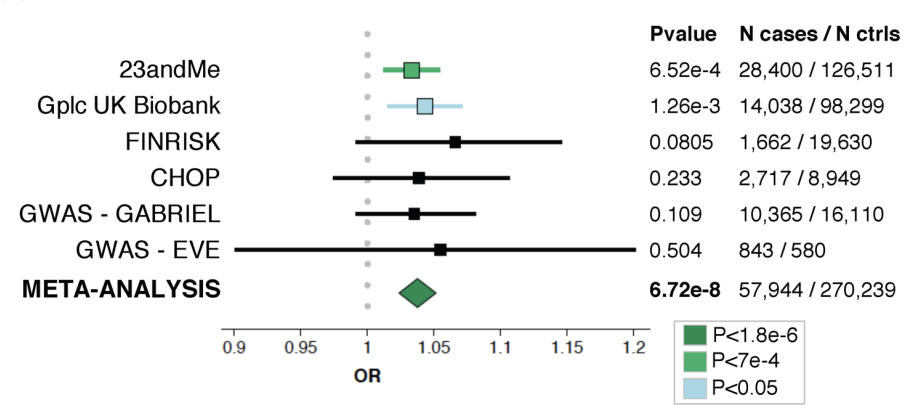

C

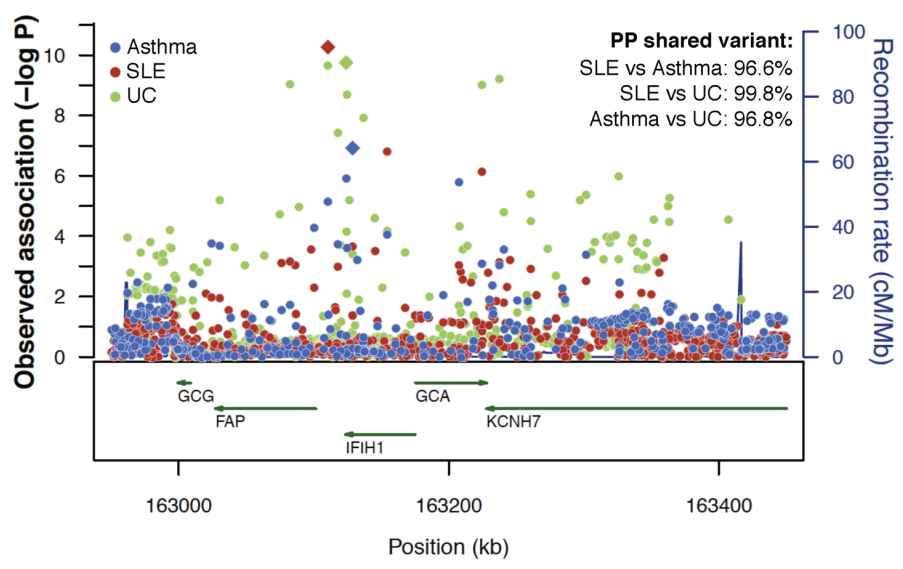

B

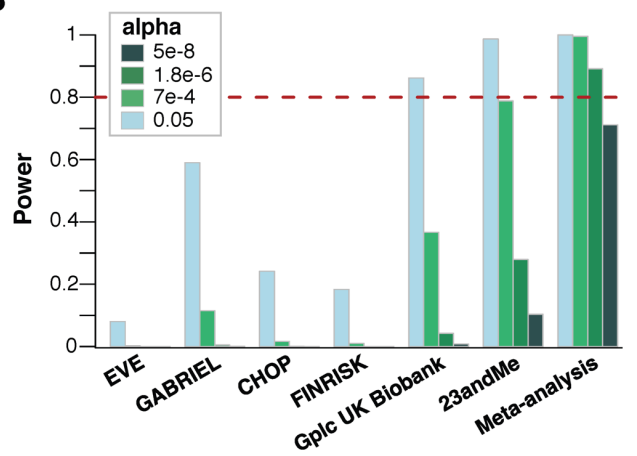

D

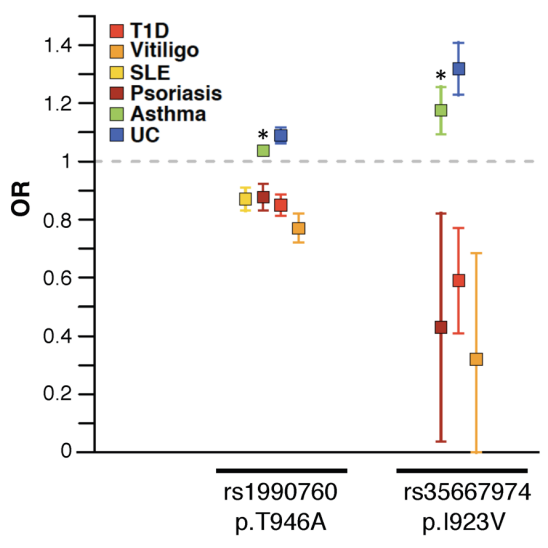

Fig. 3. Pleiotropic effects of IFIH1 LOF variants. (A) A significant association of IFIH1 rs1990760-C (p.T946A) with increased risk of asthma was observed in the meta-analysis of PheWAS and GWAS results, with consistent effect estimate across the six cohorts tested. (B) Power estimation demonstrates the lack of power to detect an association at rs1990760-C in currently available asthma GWAS studies. Power to surpass various significance cutoffs $(\mathrm{P}<0.05$; FDR $<0.1, \mathrm{P}<7 \mathrm{e}-4$; study-wide significance after Bonferroni correction, $\mathrm{P}<1.8 \mathrm{e}-6$; and genome-wide significance, $\mathrm{P}<5 \mathrm{e}-8)$ in the six cohorts was estimated using the frequency of the asthma risk allele $(\mathrm{RAF}=0.39)$, the odds ratio in the PheWAS/GWAS meta-analysis $(\mathrm{OR}=1.037)$, a disease prevalence of $8 \%$, and the number of cases and controls in each of the cohorts. (C) Co-localization analysis demonstrates that the asthma, SLE and ulcerative colitis (UC) associations at the IFIH1 locus are driven by a shared causal signal. PP, posterior probability. (D) Results from this study $(*)$ combined with previously published findings suggest an allelic series of LOF IFIHI alleles decreasing the risk of various autoimmune diseases while increasing the risk of asthma and ulcerative colitis. Association results for the reported IFIH1 loss-of-function alleles rs1990760-C (p.T946A) and rs35667974-C (p. I923V) are shown. 

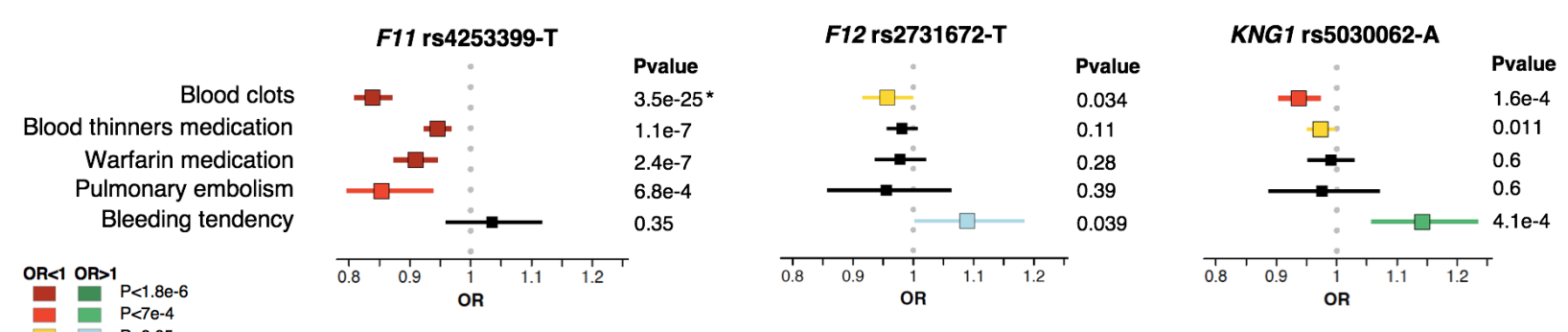

Fig. 4. PheWAS association patterns of SNPs affecting genes in the contact activation coagulation pathway. Three SNPs known to affect plasma protein levels of FXI, FXII and KNG1, and previously reported as associated with partial thromboplastin time (aPTT) were interrogated in meta-PheWAS. Five phenotypes were observed as significantly associated (FDR $<0.1)$ with at least one of the three SNPs: blood clots (known association with the F11 SNP, *), blood thinners medication, warfarin medication, pulmonary embolism, and bleeding tendency. Effects of the aPTT-increasing alleles are shown. 
Table 1. Cohorts included in this study.

\begin{tabular}{|c|c|c|c|c|}
\hline Cohort & $\begin{array}{l}\text { Participants } \\
\text { geographic } \\
\text { distribution }\end{array}$ & Phenotypes source & $\begin{array}{l}\mathrm{N} \text { binary } \\
\text { endpoints } \\
\text { tested * }\end{array}$ & $\begin{array}{l}\text { Max } \\
\text { sample } \\
\text { size } \\
\end{array}$ \\
\hline 23andMe & $89 \%$ USA (adult) & Questionnaire-based self-reports & 654 & 671,151 \\
\hline Genomics plc UK Biobank & $100 \%$ UK (adult) & $\begin{array}{l}\text { Questionnaire-based self-reports, medical } \\
\text { interviews and follow-up }\end{array}$ & 90 & 112,337 \\
\hline FINRISK & $100 \%$ Finns (adult) & National health registries (ICD8,9,10) & 278 & 21,371 \\
\hline CHOP & $\begin{array}{l}100 \% \text { USA } \\
\text { (pediatric) }\end{array}$ & Electronic health records (ICD9-CM) & 870 & 12,044 \\
\hline Genomics plc GWAS & mixed & $\begin{array}{l}\text { Mixed - multiple independent disease- } \\
\text { specific cohorts }\end{array}$ & 34 & - \\
\hline
\end{tabular}

* Number of binary endpoints with $N$ cases $\geq 20$ 
Table 2. Significant novel associations in the PheWAS meta-analysis. Associations reaching $\mathrm{P}<1.8 \mathrm{e}-6$ (Bonferroni-corrected significance threshold) in the meta-analysis of RWD PheWAS results with GWAS results are shown. The full list of potential novel SNP-phenotype pairs reaching FDR $<0.1$ is provided in Table S4. The effect of the allele increasing the risk for known associated disease(s) supporting the therapeutic hypothesis is shown. Novel associations with direction of effect opposite to the known associated disease(s) effect are highlighted in bold.

\begin{tabular}{|c|c|c|c|c|c|c|c|c|c|c|}
\hline \multirow[b]{2}{*}{ Gene } & \multirow[b]{2}{*}{ SNP } & \multirow[b]{2}{*}{ EA (EAF)* } & \multirow[b]{2}{*}{$\begin{array}{l}\text { Known Association in } \\
\text { published GWAS } \dot{\dagger}\end{array}$} & \multicolumn{7}{|c|}{ Novel Association in meta-PheWAS } \\
\hline & & & & Phenotype & OR (CI95) & Pvalue & $\begin{array}{l}\mathrm{N} \\
\text { studies }\end{array}$ & Direction $^{s}$ & $\begin{array}{l}\mathbf{N} \\
\text { cases }\end{array}$ & $\begin{array}{l}\mathbf{N} \\
\text { controls }\end{array}$ \\
\hline$C D 226$ & rs763361 & $\mathrm{T}(0.47)$ & IBD & Hypothyroidism & $1.054(1.037-1.071)$ & $8.11 \mathrm{E}-11$ & 3 & $++?+?$ & 35,428 & 412,577 \\
\hline \multirow[t]{5}{*}{ GDF15 } & rs17724992 & $\mathrm{A}(0.73)$ & BMI & Heart metabolic disease ${ }^{\wedge}$ & $1.031(1.021-1.042)$ & $3.08 \mathrm{E}-09$ & 1 & $+? ? ? ?$ & 275,944 & 209,302 \\
\hline & & & & High blood pressure $^{\wedge}$ & $1.030(1.020-1.041)$ & 7.64E-09 & 2 & $++? ? ?$ & 151,511 & 465,686 \\
\hline & & & & Blood pressure medication^ ${ }^{\wedge}$ & $1.031(1.019-1.043)$ & $1.76 \mathrm{E}-07$ & 1 & $+? ? ? ?$ & 125,406 & 394,753 \\
\hline & & & & GERD & $1.026(1.016-1.037)$ & $6.11 \mathrm{E}-07$ & 1 & $+? ? ? ?$ & 130,654 & 384,572 \\
\hline & & & & Any $\mathrm{CVD}^{\wedge}$ & $1.026(1.0 .16-1.037)$ & $1.40 \mathrm{E}-06$ & 1 & $+? ? ? ?$ & 148,577 & 388,405 \\
\hline IFIHI & rs 1990760 & $\mathrm{~T}(0.61)$ & $\mathrm{T} 1 \mathrm{D}$ & Asthma & $0.964(0.951-0.977)$ & $1.21 \mathrm{E}-07$ & 5 & ----- & 57,101 & 269,659 \\
\hline$I R F 5$ & rs 10488631 & $\mathrm{C}(0.11)$ & SLE & Hypothyroidism & $1.083(1.050-1.12)$ & $5.78 \mathrm{E}-07$ & 3 & $++?+?$ & 23,182 & 236,240 \\
\hline \multirow[t]{3}{*}{$P N P L A 3$} & rs 738409 & $\mathrm{G}(0.33)$ & ALT & Severe acne & $0.905(0.879-0.932)$ & $1.47 \mathrm{E}-11$ & 1 & $-? ? ? ?$ & 14,812 & 187,018 \\
\hline & & & & $\mathrm{T} 2 \mathrm{D}$ & $1.079(1.054-1.104)$ & $8.02 \mathrm{E}-11$ & 3 & $++? ?+$ & 51,052 & 489,421 \\
\hline & & & & High cholesterol & $0.959(0.944-0.974)$ & $1.59 \mathrm{E}-07$ & 2 & $--? ? ?$ & 101,646 & 180,947 \\
\hline \multirow[t]{2}{*}{ TYK2 } & rs34536443 & $\mathrm{G}(0.89)$ & Psoriasis & Any immune disorder & $1.101(1.071-1.131)$ & $4.27 \mathrm{E}-12$ & 1 & $+? ? ? ?$ & 112,148 & 173,986 \\
\hline & & & & Hypothyroidism & $1.138(1.080-1.198)$ & $1.19 \mathrm{E}-06$ & 3 & $++?-?$ & 23,145 & 233,757 \\
\hline
\end{tabular}

* EA, effect allele; EAF, effect allele frequency. The effect allele is the risk allele for known associated disease(s) related to the therapeutic hypothesis.

$\dagger$ Known associated disease related to the therapeutic hypothesis (surrogate for efficacy). The strongest association reported in the literature is indicated. The full list of known associations is provided in Supplementary Table 1.

${ }^{\$}$ Direction of effect in 23andMe, Genomics plc UK Biobank, FINRISK, CHOP and GWAS

ALT, alanine aminotransferase ; BMI, body mass index ; CVD, cardiovascular disease; GERD, Gastroesophageal reflux disease; IBD, inflammatory bowel disease; SLE, systemic lupus erythematosus ; T1D, type diabetes; T2D, type 2 diabetes

${ }^{\wedge}$ Correlated phenotypes 